\title{
Dynamic whole-body FDG-PET imaging for oncology studies
}

\author{
Nagara Tamaki ${ }^{1}\left[\begin{array}{l}0 \\ \text { Tomoya Kotani }^{1} \cdot \text { Motoki Nishimura }\end{array}{ }^{1} \cdot\right.$ Tomohito Kaji $^{2}$
}

Received: 30 October 2021 / Accepted: 4 January 2022 / Published online: 20 January 2022

(c) The Author(s) 2022

\begin{abstract}
Introduction Recent PET/CT systems have improved sensitivity and spatial resolution by smaller PET detectors and improved reconstruction software. In addition, continuous-bed-motion mode is now available in some PET systems for whole-body PET imaging. In this review, we describe the advantages of dynamic whole-body FDG-PET in oncology studies.

Methods PET-CT imaging was obtained at $60 \mathrm{~min}$ after FDG administration. Dynamic whole-body imaging with continuous bed motion in $3 \mathrm{~min}$ each with flow motion was obtained over 400 oncology cases. For routine image analysis, these dynamic phases (usually four phases) were summed as early FDG imaging. The image quality of each serial dynamic imaging was visually evaluated. In addition, changes in FDG uptake were analyzed in consecutive dynamic imaging and also in early delayed (90 min after FDG administration) time point imaging (dual-time-point imaging; DTPI). Image interpretation was performed by consensus of two nuclear medicine physicians.

Result All consecutive dynamic whole-body PET images of 3 min duration had acceptable image quality. Many of the areas with physiologically high FDG uptake had altered uptake on serial images. On the other hand, most of the benign and malignant lesions did not show visual changes on serial images. In the study of 60 patients with suspected colorectal cancer, unchanged uptake was noted in almost all regions with pathologically proved FDG uptake, indicating high sensitivity with high negative predictive value on both serial dynamic imaging and on DTPI. We proposed another application of serial dynamic imaging for minimizing motion artifacts for patients who may be likely to move during PET studies.

Discussion Dynamic whole-body imaging has several advantages over the static imaging. Serial assessment of changes in FDG uptake over a short period of time is useful for distinguishing pathological from physiological uptake, especially in the abdominal regions. These dynamic PET studies may minimize the need for DPTI. In addition, continuous dynamic imaging has the potential to reduce motion artifacts in patients who are likely to move during PET imaging. Furthermore, kinetic analysis of the FDG distribution in tumor areas has a potential for precise tissue characterization.

Conclusion Dynamic whole-body FDG-PET imaging permits assessment of serial FDG uptake change which is particularly useful for differentiation of pathological uptake from physiological uptake with high diagnostic accuracy. This imaging can be applied for minimizing motion artifacts. Wide clinical applications of such serial, dynamic whole-body PET imaging is expected in oncological studies in the near future.
\end{abstract}

Keywords Whole-body PET $\cdot{ }^{18} \mathrm{~F}-\mathrm{FDG} \cdot$ Dynamic acquisition $\cdot$ Cancer

\section{Introduction}

${ }^{18} \mathrm{~F}$-FDG-PET/CT is now widely used as a valuable imaging modality in clinical oncology. This is commonly used for tumor characterization, staging, restaging, and therapy monitoring [1-5]. FDG-PET imaging has been used as sur-

Nagara Tamaki

natamaki@koto.kpu-m.ac.jp

1 Department of Radiology, Graduate School of Medical Science, Kyoto Prefectural University of Medicine, Kyoto, Japan

2 Hakodate Goryokaku Hospital, Hakodate, Japan rogate for assessment of tumor glycolytic activity, which is an important marker of tumor biology. Current FDG wholebody PET/CT imaging is performed at a predefined time point, usually $60 \mathrm{~min}$ after tracer administration. PET/CT images are reviewed qualitatively for FDG uptake (positive 
or negative) and semi-quantitative measure of glucose uptake by the standardized uptake value (SUV) [6-8]. On the other hand, FDG uptake is also seen in physiological tissues. Thus, it is often difficult to differentiate pathological ${ }^{18} \mathrm{~F}$-FDG uptake from physiological ${ }^{18} \mathrm{~F}$-FDG uptake in routine static PET imaging [9-15].

Present study summarizes advantages of serial dynamic whole-body PET imaging and introduces several clinical values of assessing dynamic FDG uptake changes in oncology studies.

\section{Methods}

Most PET systems use step-and-shoot imaging for wholebody imaging, in which dynamic PET study may be confined to a single-bed position. Some new PET camera systems have continuous-bed-motion mode for whole-body imaging using flow-motion system [16-18]. This dynamic wholebody imaging has several advantages over the conventional step-and-shoot bed motion (Fig. 1). Actual field of view is the area of detector field of view in step-and-shoot motion which may show low sensitivity in peripheral areas of view. Thus, some overlap imaging is needed for each step for whole-body imaging. In continuous bed motion, on the other hand, the sensitivity over the axial range is uniform with requiring no overlap [16-18]. Therefore, the whole-body imaging with continuous bed motion permits better uniformity, reproducibility, and higher sensitivity than the conventional whole-body imaging with step-and-shoot motion. In addition, FDG uptake change and motion may be visually assessed using various sequential analysis (Table 1).

In our PET centers, each patient fasted for $>4 \mathrm{~h}$, and their blood glucose levels were below $200 \mathrm{mg} / \mathrm{dl}$ prior to FDG injection. FDG-PET/CT imaging was obtained at $60 \mathrm{~min}$ after administration of $2-5-\mathrm{MBq} / \mathrm{kg}$ body weight of ${ }^{18}$ F-FDG. We performed serial dynamic whole-body imaging with continuous bed motion at varying speeds $(6 \mathrm{~mm} / \mathrm{s}$ from the head to the pelvis and $14 \mathrm{~mm} / \mathrm{s}$ for the lower limbs in our hospital) to acquire each whole-body phase in $\sim 3$ min in list mode using Biograph Horizon with flow motion (Siemens Medical Solutions) [19, 20].
Fig. 1 Actual field of view on step-and-shoot imaging and continuous-bed-motion imaging. Since sensitivity is low in the peripheral areas of view, and thus, some overlap imaging is needed for whole-body imaging in step-and-shoot imaging. On the other hand, actual field of view is uniform without need for overlap imaging in continuous bed motion. Thus, the sensitivity is higher and uniform for whole-body imaging in the continuous bed motion
Table 1 Advantages of dynamic whole-body imaging using continuous bed motion

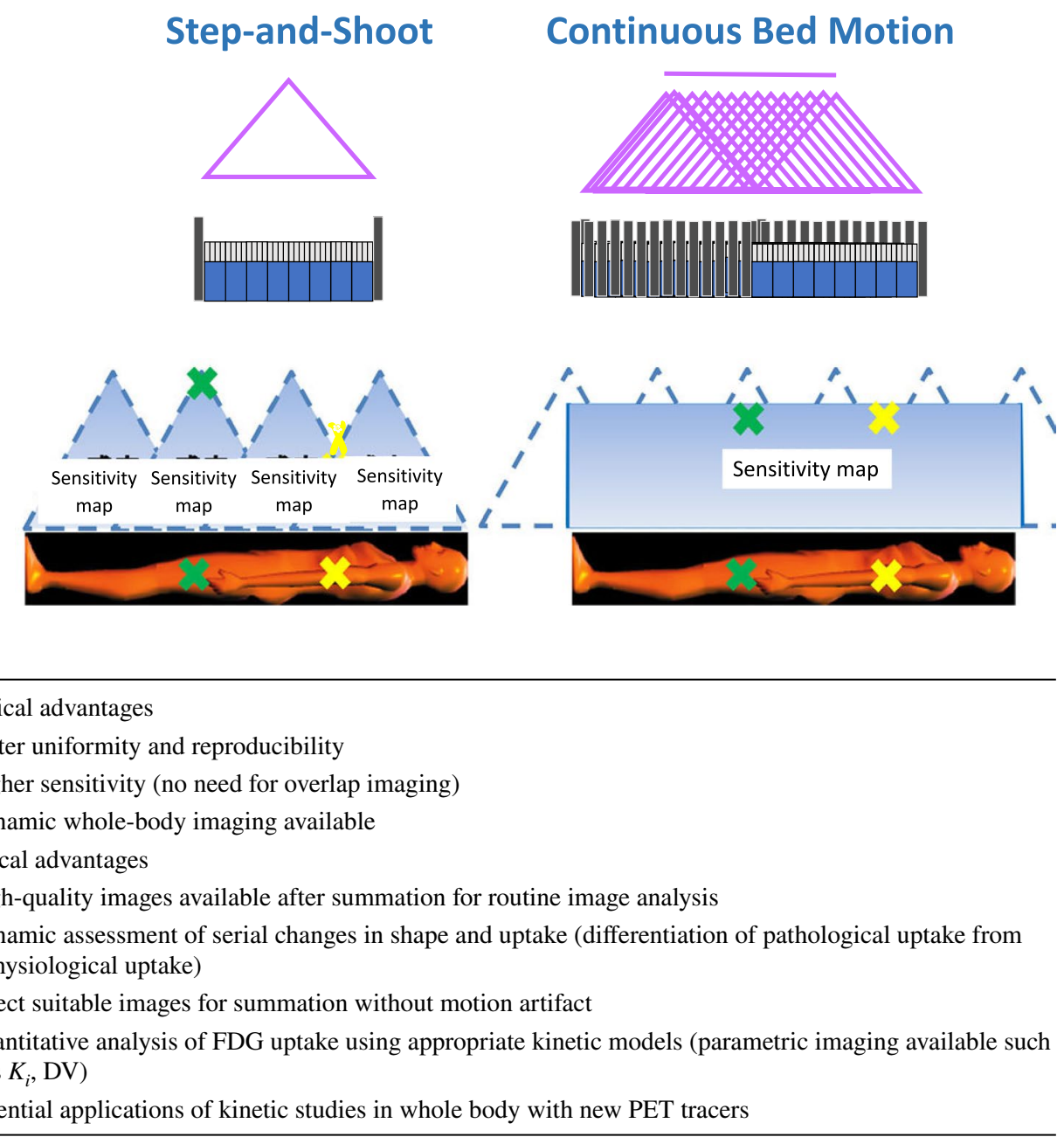


For the purpose of routine image interpretation, these dynamic phases (usually four phases) were summed in a reconstruction equivalent to a 12-min whole-body acquisition for routine image interpretation. The dynamic whole-body PET images and the summed whole-body PET image were reconstructed using attenuation-corrected ordinary Poisson three-dimensional ordered-subset expectation maximization (3D-OSEM) reconstruction with a 4-mm post-reconstruction Gaussian filter, using a $180 \times 180$-pixel matrix [19, 20],

Image interpretation was performed by two board-certified nuclear medicine physicians and the final judgment was decided by consensus [19,20].

\section{Results}

\section{Image quality}

We have acquired over 400 cases of this dynamic wholebody FDG-PET imaging as a part of routine procedures, particularly for patients suspected with abdominal areas. Two board-certificated nuclear medicine physicians performed image interpretation and assessed the image quality of the serial dynamic whole-body and summed images $[19,20]$. All of the 3-min serial dynamic whole-body PET images were of acceptable image quality. Most of 12-min summed whole-body images provided high-quality images which are commonly used for routine image interpretation [19, 20]. In addition, dynamic whole-body imaging has been used for various clinical purposes as described in Table 1.

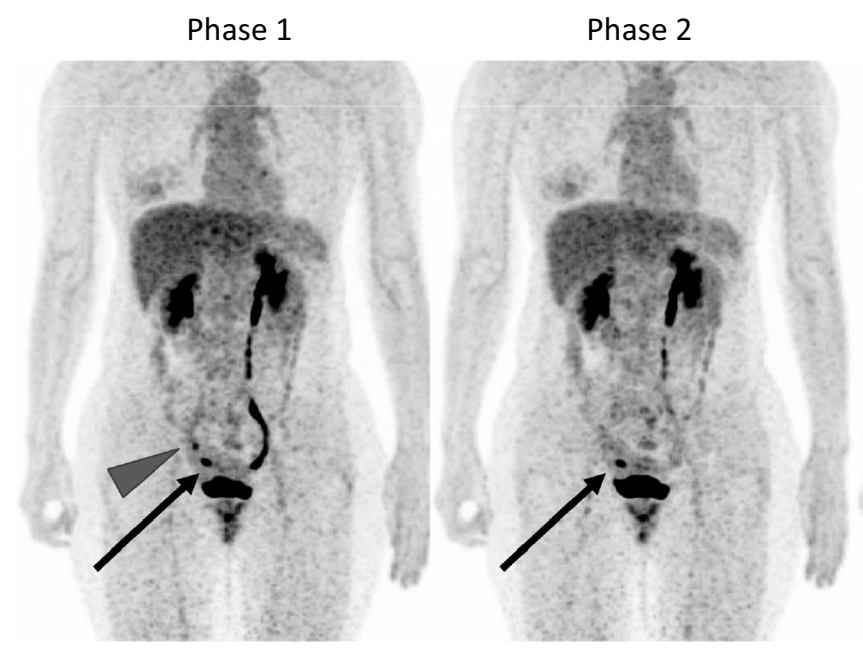

\section{Differentiation of pathological uptake from physiological uptake}

It is often difficult to differentiate pathological ${ }^{18} \mathrm{~F}-\mathrm{FDG}$ uptake from physiological ${ }^{18} \mathrm{~F}-\mathrm{FDG}$ uptake on routine static PET imaging. Dynamic distribution of ${ }^{18} \mathrm{~F}-\mathrm{FDG}$ uptake may hold a promise to differentiate pathological uptake without change from physiological uptake with change (Table 1). Accordingly, we performed dynamic whole-body imaging to assess the serial change of ${ }^{18} \mathrm{~F}$-FDG uptake in various anatomical regions. Areas of high ${ }^{18}$ F-FDG uptake were identified in dynamic images and summed image. In dynamic image evaluation, if an area of high uptake disappeared in any phase, or if more than half of the area of uptake showed morphologic change, these were defined as areas of "changed uptake".

In dynamic image evaluation, physiological changed uptake was found in $9 / 19$ areas in gastric region (47\%), $32 / 39$ areas in small intestine (82\%), 17/33 areas in colon (52\%), and 60/63 areas in ureter (95\%) (Fig. 2). On the other hand, most of benign and malignant lesions showed no visual change in serial images, suggesting high diagnostic value for differentiating physiological FDG uptake from pathological FDG uptake based on presence of uptake change on serial dynamic imaging [19].

A number of studies indicate that malignant lesions often show a gradual increase of FDG accumulation from an early phase to a delayed phase, and therefore, dualtime-point imaging (DTPI) may provide increased ability to detect malignant lesions and facilitate differentiation of malignant lesions from benign lesions [21-27]. Thus, delayed (90-120 min after FDG administration) PET imaging is often applied to compare the FDG uptake with that on conventional early (60 min after FDG administration)
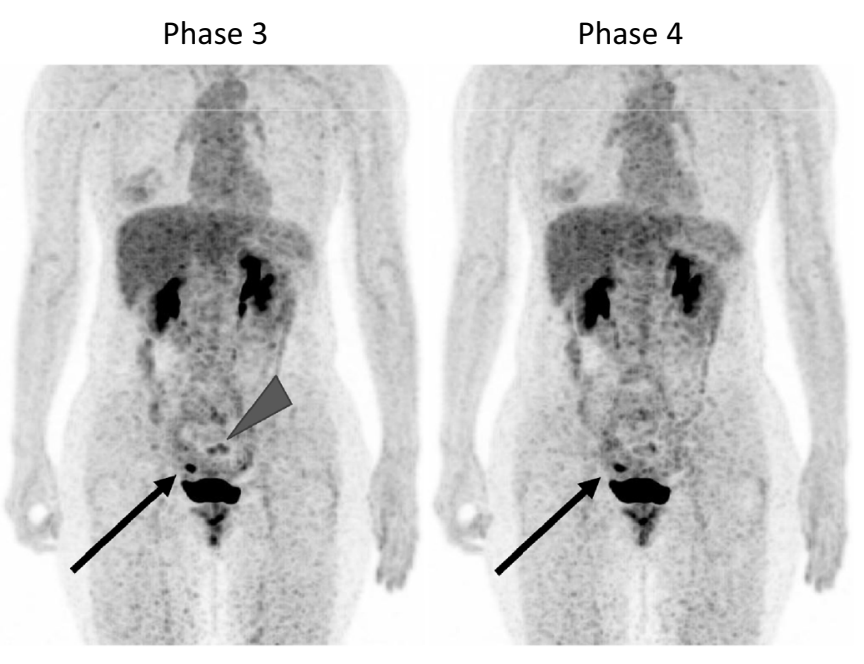

Fig. 2 Four serial whole-body dynamic PET images ( 3 min each) of a patient with rectal cancer. FDG uptake in the rectal cancer does not move (arrows) but other FDG uptakes in ureter and sigmoid colon move (arrow heads) 
PET imaging. This early delayed imaging (dual-time-point imaging; DTPI) plays a valuable role for the differentiating FDG take lesions [21-27]. However, such delayed imaging is cumbersome for patients who have to wait to come back to PET room for separate delayed imaging. In addition, such imaging requires additional machine time and another radiation burden to each patient by additional $\mathrm{CT}$ for attenuation correction.

We assessed the frequency of change observed in colorectal uptake shape by dynamic PET imaging as compared to DTPI, to differentiate pathological uptake from physiological uptake in 60 patients with suspected colorectal cancer [20]. Particularly, when changed FDG uptake was seen on serial dynamic imaging as well as DTPI, such lesion was highly likely to represent physiological FDG uptake. Among 73 histologically proven pathological FDG uptakes, unchanged uptake was noted in almost all regions with high sensitivity on both serial dynamic imaging and on DTPI (Fig. 3). In addition, most of the changed FDG uptake on either serial dynamic imaging or DTPI was physiological uptake, indicating very high negative predictive value. These results indicated that high focal FDG uptake was highly likely to be physiological uptake when the focal FDG uptake showed changes on serial dynamic imaging and DTPI, as well [20]. Our results also indicated slightly but significantly higher diagnostic accuracy of serial dynamic imaging for such differentiation than DTPI.

Similar results were obtained with other abdominal cancer, such as gynecological tumors (Fig. 4). Based on these results, serial dynamic imaging permits assessment of sequential changes in uptake shape within a short period without any additional imaging time.

\section{Minimizing motion artifact}

Typical patient motion has several types, including periodic respiratory and cardiac motion, non-periodical motion of the stomach and bowel system, and bulk body motion [28-30]. The most straightforward correction method is to perform respiratory and electrocardiographic gated
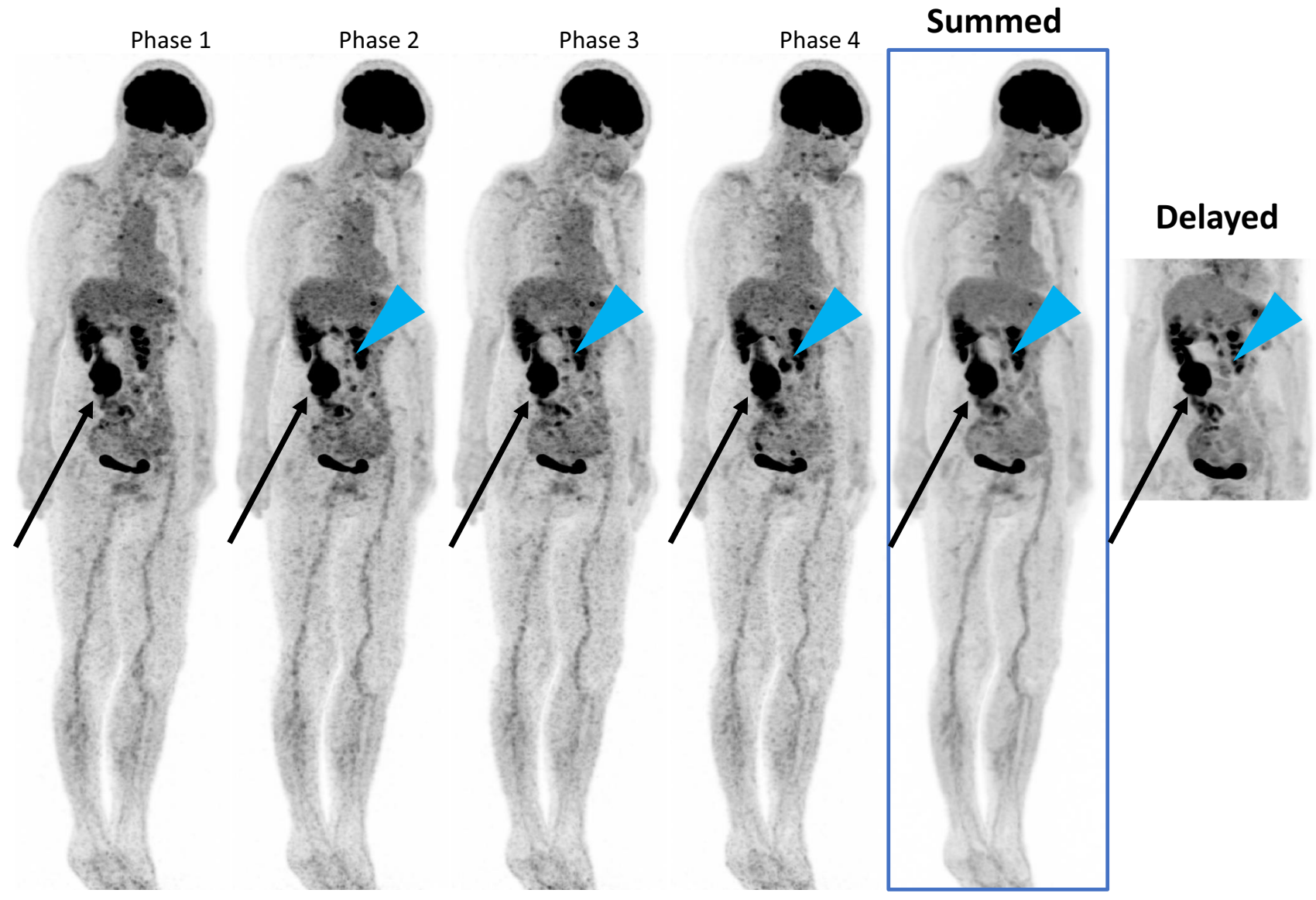

Fig. 3 Four serial whole-body dynamic PET images (3 min each), the summed image (12 min), and the delayed image of a patient with colon cancer. High FDG uptake in the ascending colon does not move (arrows), but adjacent FDG uptake in colon changes in shape (arrow

heads) on serial dynamic imaging. The FDG uptake change is also noted on summed early and delayed imaging, but such FDG uptake change is better identified by serial dynamic imaging 


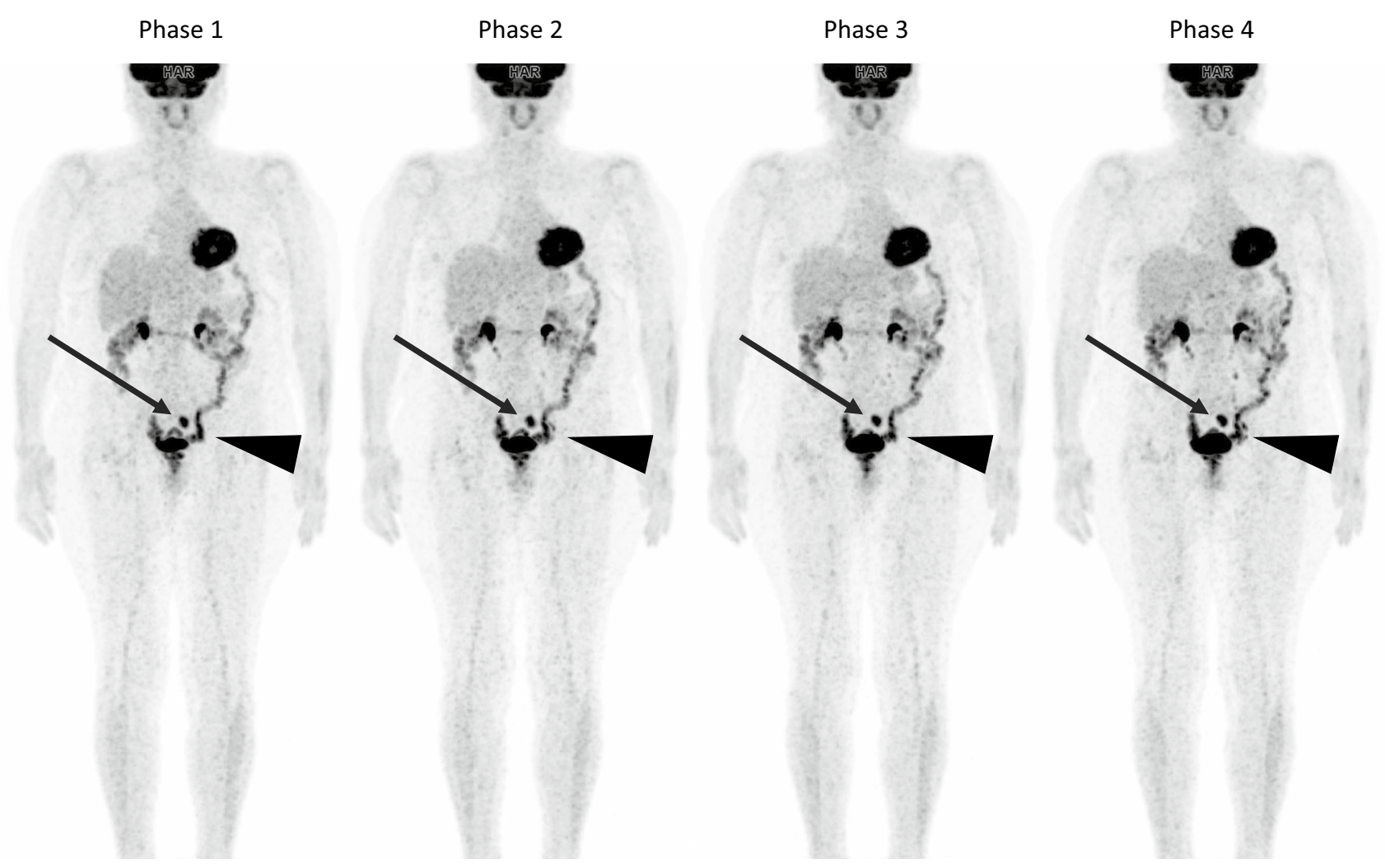

Fig. 4 Four serial whole-body dynamic PET images ( 3 min each) of a patient with endometrial cancer. FDG uptake in the endometrial cancer does not move (arrows) but other FDG uptakes in sigmoid colon slightly move (arrow heads)

acquisition, to accept only data from the end-expiration phase of the breathing cycle or end-diastolic phase of the cardiac cycle. The motion noise may be minimized with short PET acquisition. However, medication may reduce bowel motion for imaging.

Actual patient motion should seriously be considered for appropriate PET imaging. Since most FDG-PET-CT studies are performed for oncology patients, many of them may have difficulty in staying still without motion during PET/ CT imaging due to pain. It is also difficult to ask young child or demented person to stay still for imaging. Patient motion may often create detrimental motion artifacts causing difficulty in image interpretation. When the patient motion is simple, such motion may be corrected by use of anatomical information for suitable reconstruction [31]. However, complex motion may cause difficulties in motion correction in many cases.

We have proposed another application of serial wholebody dynamic imaging for minimizing motion artifacts. Figure 5 shows serial 3-min dynamic whole-body images of 82-year-old patient with lung cancer. The Phase 3-to-Phase 5 images suggested arm motions. While summation of Phase 1 through Phase 5 images showed striking motion artifacts in chest and arms, such motion artifacts were minimized by summation of Phase 1 and Phase 2 images. In addition, the image quality was high with these summation as compared to the individual 3-min whole-body images [32].

\section{Potential roles for quantitative assessment}

PET has a great advantage for quantitative analysis of tracer concentration and metabolic alterations using suitable tracer kinetic models. FDG uptake and clearance depend on the time interval between intravenous FDG administration and imaging. On sequential image analysis after FDG administration, tissues with high glycolysis may have continuously increasing amounts of FDG trapped in cells in the form of FDG-6-phosphate, while FDG uptake may decrease over time in tissues with high glucose-6-phosphatase. In many PET studies, standardized uptake (SUV) value of FDG at the time of FDG-PET imaging is quantitatively analyzed and displayed. In addition, SUV uptake change over time is analyzed over 30-60 min using DTPI in various oncology fields [33-40]. On the basis of the concept of differences in glucose metabolism, FDG uptake tends to increase or remain high in many malignant lesions as compared to benign lesions including infection and inflammation. Such differences may be nicely assessed by sequential FDG imaging. However, a number of other studies have demonstrated significant overlap of FDG uptake patterns between benign and 


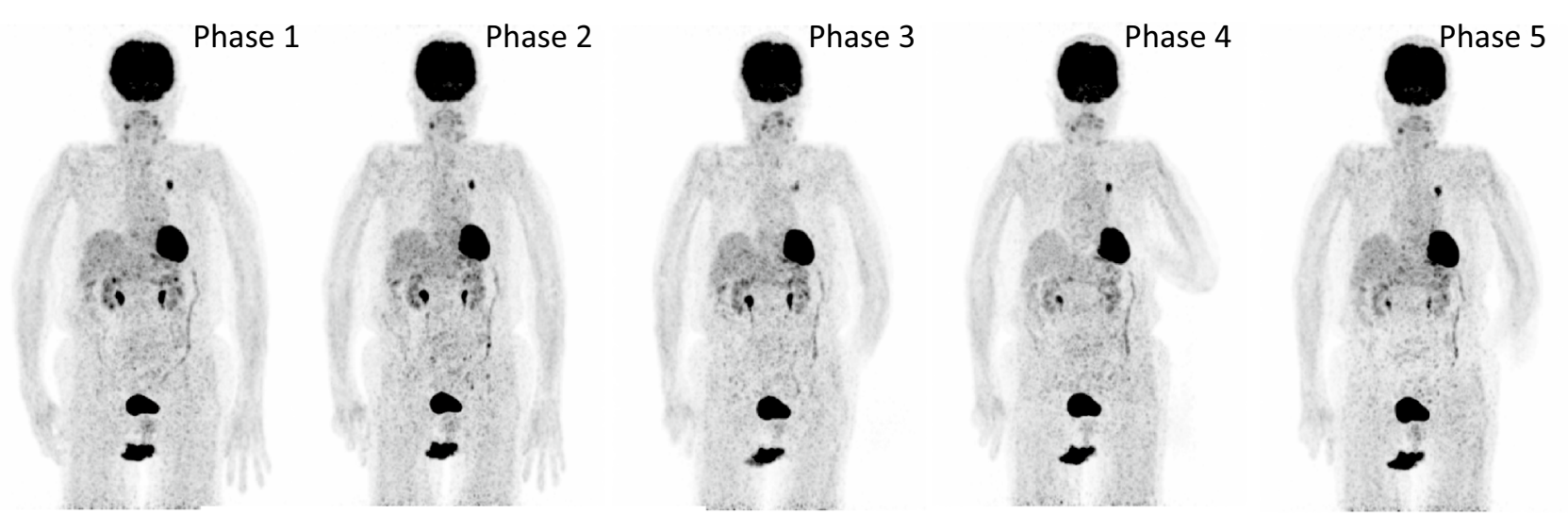

Summation of Phase $1 \& 2$

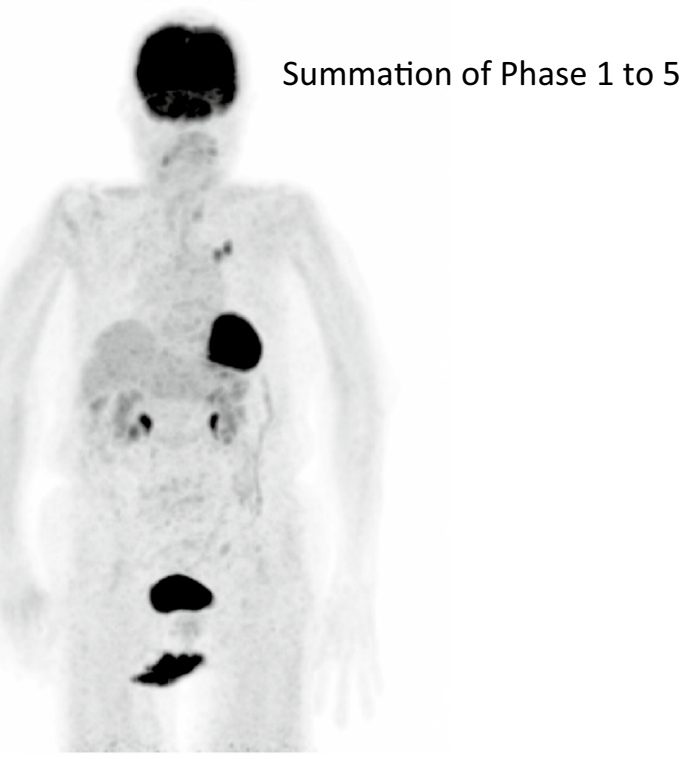

Fig. 5 Five serial dynamic whole-body PET images (3 min each) (top) of 82-year-old patient with lung cancer. This patient moved during Phase 3 through Phase 5 images. The summed-up FDG image (from Phase 1 to Phase 5) (bottom right) shows motion artifact,

malignant lesions even on DTPI [33, 41]. Serial dynamic whole-body PET also enables semi-quantitative analysis of FDG uptake changes similarly with DTPI (Fig. 6) [18]. Serial imaging has advantages for more reliable assessment of FDG uptake change using multiple points as compared to the two-point analysis with DTPI (Table 1).

\section{Discussion}

Dynamic whole-body imaging has several advantages over a single static imaging. Serial assessment of changes in FDG uptake over a short period of time is useful for distinguishing pathological from physiological uptake. It is effective in distinguishing pathological from physiological uptake, especially in the abdominal region [19]. including pulmonary lesion. However, the motion artifact is minimized with better image quality on the selected summed-up image (Phase 1 and Phase 2) (bottom left)

These dynamic PET studies may minimize the need for DPTI [20]. In addition, continuous dynamic imaging has the potential to reduce motion artifacts in patients who are likely to move during PET imaging. The total acquisition time for this dynamic whole-body imaging (3 min $\times 4-5$ ) is same as conventional single whole-body imaging (12-15 min). The major differences are that this dynamic imaging may require small additional time for reconstruction and data management as compared a single whole-body imaging.

These conclusions were obtained for those suspected with colorectal cancer and other abdominal cancer, as shown in Fig. 4. This new imaging is particularly useful to differentiate para-aortic or para-iliac lymph-node involvements from physiological FDG uptake in ureter, colon, or ovaries. Furthermore, this new imaging may be able to minimize 


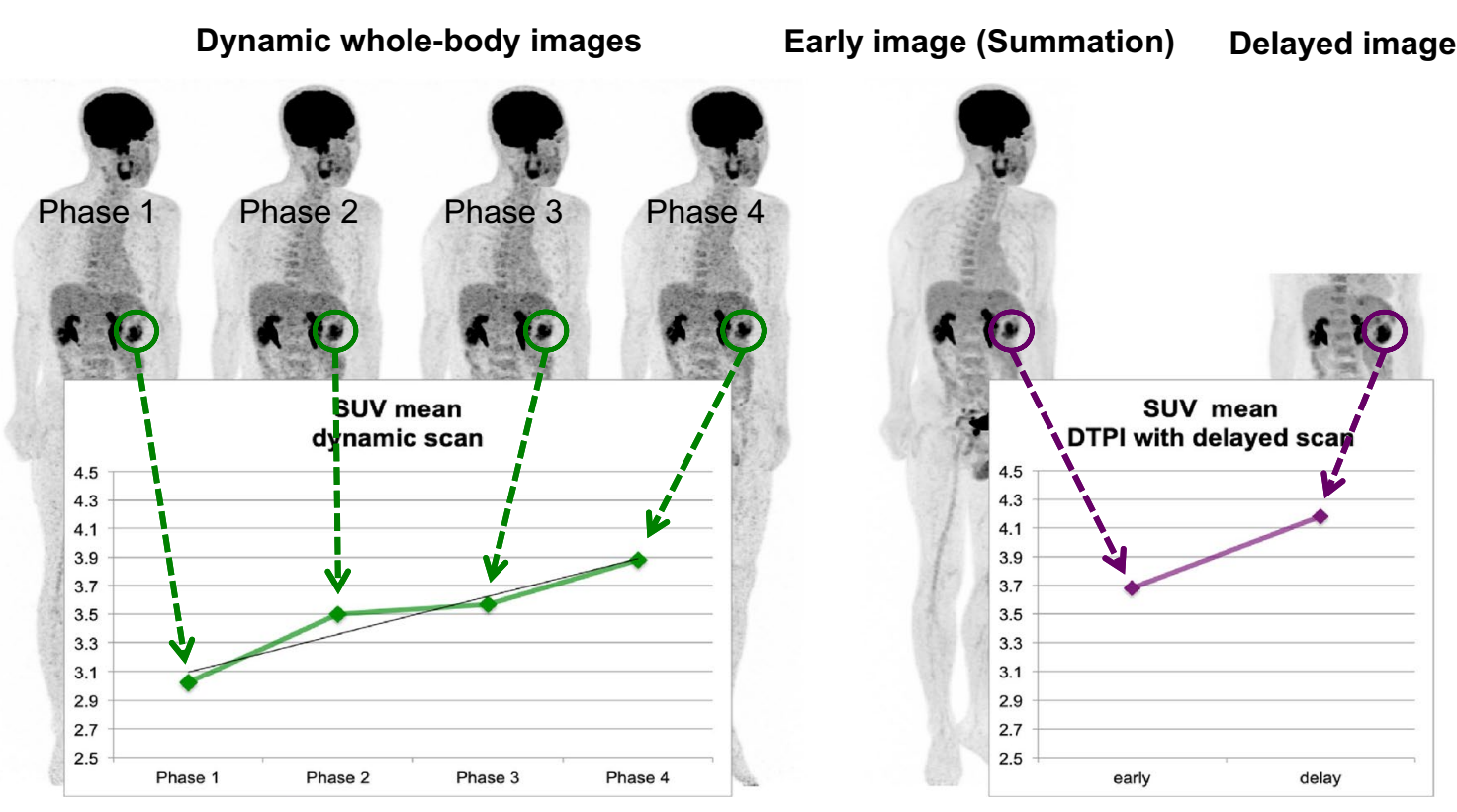

Fig. 6 Four serial dynamic whole-body PET images (left) and early (summation)/delated images (right) of a patient with colon cancer. High and persistent FDG uptake is noted (circles) with FDG count

the need of delayed imaging for assessment of FDG uptake change.

There are several reasons why the serial dynamic imaging showed a better FDG uptake in motion than DTPI. FDG may accumulate in the wall of the GI tract but may also be secreted inside the GI tract, resulting in to-and-fro motion in the GI tract [42, 43]. Dynamic whole-body imaging has the additional advantage of enabling the assessment of uptake change over time on multiple serial images (sometimes with cine-mode display) as compared to only two images on DTPI.

There seems to be rather rare to show significant artifacts due to patient motion during PET study. In addition, a striking patient motion may stop the image acquisition to reset the PET camera for another imaging. However, such serial dynamic imaging is particularly useful in reducing mild but significant motion artifacts for patients who may be likely to move during PET studies (Table 1).

Another important aspect of this dynamic imaging is for semi-quantitative analysis of FDG uptake changes. We analyzed count change in SUV mean by making small region of interest (ROI) in abnormal uptake areas as compared to the early delayed imaging, as shown in Fig. 6.

New strategies are developed for the generation of parametric images (pixel-by-pixel analysis), ranging from graphical analysis, such as Patlak method [44, 45]. The graphical analysis is a simple, robust, and enables the direct estimation of the primary kinetic macro-components of the tracer uptake across multiple fields of views. The serial dynamic increase in the lesion on dynamic images. Such FDG uptake increase is also noted on dual-time-point imaging (DTPI)

whole-body PET imaging is considered as a suitable method for this purpose. Recently, there are a number of reports which indicate clinical feasibility of dynamic FDG-PET/CT applied on a pilot cohort of oncology cases [46-48]. One of the preliminary studies indicates that multi-pass whole-body PET $K_{i}$ parametric imaging utilizing robust Patlak graphical analysis may achieve equivalent or, potentially, superior lesion detectability than standard-of-care SUV imaging with reduced false-positive rates in routine oncology applications [48]. $K_{i}$ parametric imaging seems to be particularly valuable to differentiate abnormal uptake lesions with gradual increase in FDG uptake from physiological uptake areas with gradual decrease in uptake such as liver and vessels. $K_{i}$ may reflect count change, and, therefore, has a potential to enhance detection of abnormal FDG uptake lesions in high background areas [48, 49].

The Patlak model is valid after some time when the free tracer has reached a steady state between blood and tissue. This model estimates the Patlak slope $\left(K_{i}\right)$ which is the rate of irreversible uptake, and the Patlak intercept (DV), which is the apparent distribution volume of non-metabolized tracer (Table 1). Accordingly, the metabolic rate of FDG is estimated as follows: $\mathrm{MRFDG}=K_{i} \times$ blood glucose $[49,50]$.

One of the major issues for kinetic analysis is how to obtain suitable input function for graphic analysis. Arterial or arterialized venous blood sampling is commonly performed particularly for brain studies, but this is quite invasive [44, 51-53]. Image-based input function can be obtained by serial dynamic PET imaging which covers large 
arterial blood regions such as the left ventricle and aorta [54]. Recently, standardized input function was proposed as a surrogate which may facilitate such parametric studies, as compared to the actual measurement of input function on rapid dynamic imaging in the cardiac areas [55-58]. Such precise FDG kinetic analysis seems rather complicated and remains investigative. However, serial dynamic FDG-PET imaging may hold a new promise for quantitative analysis of glucose metabolism with suitable kinetic models for previse tissue characterization in variety fields, including oncology area.

\section{Conclusions and future directions}

Recent advances of PET camera system permit dynamic whole-body imaging. This new imaging has significant advantages over the conventional step-and-shoot bed motion. Serial assessment of FDG uptake changes within short time interval is valuable in differentiating pathological uptake from physiological uptake, particularly in abdominal areas. Such serial dynamic imaging is useful to reduce possible mild but significant motion artifacts for patients who may highly likely to move during PET studies. Furthermore, more precise assessment of tissue characterization is possible in oncological studies. Such dynamic tracer kinetic studies are needed in the whole-body areas using new PET tracers (Table 1). Since such elegant image acquisition and analysis will become available with new and high-performance PET systems, wide clinical applications of such imaging are expected in the near future.

Acknowledgements We appreciate Siemens Medical Systems for strong technical support for imaging and reconstruction.

\section{Declarations}

Conflict of interest All authors declare that there has no conflict of interest.

Open Access This article is licensed under a Creative Commons Attribution 4.0 International License, which permits use, sharing, adaptation, distribution and reproduction in any medium or format, as long as you give appropriate credit to the original author(s) and the source, provide a link to the Creative Commons licence, and indicate if changes were made. The images or other third party material in this article are included in the article's Creative Commons licence, unless indicated otherwise in a credit line to the material. If material is not included in the article's Creative Commons licence and your intended use is not permitted by statutory regulation or exceeds the permitted use, you will need to obtain permission directly from the copyright holder. To view a copy of this licence, visit http://creativecommons.org/licenses/by/4.0/.

\section{References}

1. Juweid ME, Cheson BD (2006) Positron-emission tomography and assessment of cancer therapy. N Engl J Med 354:496-507

2. Weber WA (2006) Positron emission tomography as an imaging biomarker. J Clin Oncol 24:3282-3292

3. Fletcher JW, Djulbegovic B, Soares HP, Siegel BA, Lowe VJ, Lyman GH, Coleman RE, Wahl R, Paschold JC, Avril N, Einhorn LH, Suh WW, Samson D, Delbeke D, Gorman M, Shields AF (2008) Recommendations on the use of ${ }^{18}$ F-FDG PET in oncology. J Nucl Med 49:480-508

4. El-Galaly TC, Gormsen LC, Hutchings M (2017) PET/CT for staging: past, present, and future. Semin Nucl Med 48:4-16

5. Wahl RL, Jacene H, Kasamon Y, Lodge MA (2009) From RECIST to PERCIST: evolving considerations for PET response criteria in solid tumors. J Nucl Med 50(Suppl 1):122s-s150

6. Keyes JW Jr (1995) SUV: standard uptake or silly useless value? J Nucl Med 36:1836-1839

7. Thie JA (2004) Understanding the standardized uptake value, its methods, and implications for usage. J Nucl Med 45:1431-1434

8. Boellaard R, Krak NC, Hoekstra OS, Lammertsma AA (2004) Effects of noise, image resolution, and ROI definition on the accuracy of standard uptake values: a simulation study. J Nucl Med 45:1519-1527

9. Davidson T, Komisar O, Korach J, Felder S, Apter S, Ben-Haim S, Perri T (2018) Physiologic uptake of ${ }^{18}$ F-FDG in transposed ovaries may mimic metastasis on 18F-FDG PET/CT imaging. Nucl Med Commun 39(2):171-178

10. Parida GK, Roy SG, Kumar R (2017) FDG-PET/CT in skeletal muscle: pitfalls and pathologies. Semin Nucl Med 47(4):362-372

11. Rosenbaum SJ, Lind T, Antoch G, Bockisch A (2006) Falsepositive FDG PET uptake-the role of PET/CT. Eur Radiol 16(5):1054-1065

12. Lakhani A, Khan SR, Bharwani N, Stewart V, Rockall AG, Khan S, Barwick TD (2017) FDG PET/CT pitfalls in gynecologic and genitourinary oncologic imaging. Radiographics 37(2):577-594

13. Sena Y, Matsumoto S, Silman C, Otsuka K, Kiyota T (2020) Physiological 18F-FDG uptake in the normal adult anal canal: evaluation by PET/CT. Ann Nucl Med 34(8):538-544

14. Lee N, IR Y, Park SY, Yoon H, Lee Y, Oh JK (2015) Significance of incidental nasopharyngeal uptake on (18)F-FDG PET/ CT: patterns of benign/physiologic uptake and differentiation from malignancy. Nucl Med Mol Imaging 49(1):11-8

15. Nakamura $S$, Okochi K, Murata Y, Shibuya H, Kurabayashi $T$ (2009) [18F]Fluorodeoxyglucose-PET/CT differentiation between physiological and pathological accumulations in head and neck. Nucl Med Commun 30(7):498-503

16. Dahlbom M, Reed J, Young J (2001) Implementation of true continuous bed motion in 2-D and 3-D whole-body PET scanning. IEEE Trans Nucl Sci 48(4):1465-1469

17. Osborne DR, Acuff S, Cruise S, Syed M, Neveu M, Stuckey A, Bradley Y (2014) Quantitative and qualitative comparison of continuous bed motion and traditional step and shoot PET/CT. Am J Nucl Med Mol Imaging 5(1):56-64

18. Rahmim A, Lodge MA, Karakatsanis NA, Panin VY, Zhou Y, McMillan A, Cho S, Zaidi H, Casey ME, Wahl RL (2019) Dynamic whole-body PET imaging: principles, potentials and applications. Eur J Nucl Med Mol Imag 46:501-18

19. Nishimura M, Tamaki N, Matsushima S, Yamada S, Nii T, Domoto H, Yamada K (2019) Uptake changes on the whole-body dynamic ${ }^{18} \mathrm{~F}$-FDG PET may assess tissue characterization. Comparison with the conventional delayed scan. J Nucl Med 60:1284

20. Kotani T, Nishimura M, Tamaki N, Matsushima S, Akiyama S, Kanayama T, Bamba C, Tanada Y, Nii T, Yamada K (2021) Comparison between dynamic whole-body FDG-PET and 
early-delayed imaging for the assessment of motion in focal uptake in colorectal area. Ann Nucl Med 35(12):1305-1311 (in press)

21. Nishiyama Y, Yamamoto Y, Fukunaga K, Kimura N, Miki A, Sasakawa $Y$ et al (2006) Dual-time-point ${ }^{18} \mathrm{~F}$-FDG PET for the evaluation of gallbladder carcinoma. J Nucl Med 47:633-638

22. Caprio MG, Cangiano A, Imbriaco M, Soscia F, Di Martino G, Farina A et al (2010) Dual-time-point [18F]-FDG PET/CT in the diagnostic evaluation of suspicious breast lesions. Radiol Med 115:215-224

23. Tian R, Su M, Tian Y, Li F, Li L, Kuang A et al (2009) DualtimePET/CT with F-18 FDG for the differentiation of malignant and benign bone lesions. Skeletal Radiol 38:451-458

24. Nakamoto Y, Higashi T, Sakahara H, Tamaki N, Kogire M, Doi $\mathrm{R}$ et al (2000) Delayed (18)F-fluoro-2-deoxy-D-glucose positron emission tomography scan for differentiation between malignant and benign lesions in the pancreas. Cancer 89:2547-2554

25. Miyake KK, Nakamoto Y, Togashi K (2012) Dual-time-point 18FFDG PET/CT in patients with colorectal cancer: clinical value of early delayed scanning. Ann Nucl Med 26(6):492-500

26. Minamimoto R, Terauchi T, Jinnouchi S, Yoshida T, Tsukamoto E, Shimbo T, Ito K, Uno K, Ohno H, Oguchi K, Kato S, Kaneko K, Satoh Y, Tamaki T, Nakahara T, Morooka M, Inoue T, Senda M (2013) Observer variation study of the assessment and diagnosis of incidental colonic FDG uptake. Ann Nucl Med 27(5):468-477

27. Zade A, Purandare N, Rangarajan V, Shah S, Agarwal A, Kulkarni $M$ et al (2012) Role of delayed imaging to differentiate intense physiological 18F FDG uptake from peritoneal deposits in patients presenting with intestinal obstruction. Clin Nucl Med 37(8):783-785

28. Pépin A, Daouk J, Bailly P, Hapdey S, Meyer ME (2014) Management of respiratory motion in PET/computed tomography: the state of the art. Nucl Med Commun 35(2):113-122

29. Slomka PJ, Pan T, Germano G (2016) Recent advances and future progress in PET instrumentation. Semin Nucl Med 46(1):5-19

30. Gillman A, Smith J, Thomas P, Rose S, Dowson N (2017) PET motion correction in context of integrated PET/MR: current techniques, limitations, and future projections. Med Phys 44(12):e430-e445

31. Chun SY (2016) The use of anatomical information for molecular image reconstruction algorithms: attenuation/scatter correction, motion compensation, and noise reduction. Nucl Med Mol Imaging 50(1):13-23

32. Kaji T, Osanai K, Nakata T, Tamaki N (2020) Dynamic wholebody ${ }^{18} \mathrm{~F}$-FDG PET for minimizing patient motion artifact. Clin Nucl Med 45(11):880-882

33. Parghane RV, Basu S (2017) Dual-time point ${ }^{18}$ F-FDG-PET and PET/CT for differentiating benign from malignant musculoskeletal lesions. Semin Nucl Med 47(4):373-391

34. Park S, Paeng JC, Kang CH, Cheon GJ, Kang KW, Chung JK, Lee DS (2018) Dual-time point ${ }^{18}$ F-FDG PET/CT for the staging of oesophageal cancer: the best diagnostic performance by retention index for $\mathrm{N}$-staging in non-calcified lymph nodes. Eur J Nucl Med Mol Imaging 45(8):1317-1328

35. Sasada S, Masumoto N, Kimura Y, Kajitani K, Emi A, Kadoya T, Okada M (2019) Identification of axillary lymph node metastasis in patients with breast cancer using dual-phase FDG PET/CT. AJR Am J Roentgenol 213(5):1129-1135

36. Okazaki E, Seura H, Hasegawa Y, Okamura T, Fukuda H (2019) Prognostic value of the volumetric parameters of dualtime-point ${ }^{18}$ F-FDG PET/CT in non-small cell lung cancer treated with definitive radiation therapy. AJR Am J Roentgenol 213(6): 1366-1373

37. Bieg C, Mongelli F, Peterli R, Chirindel AF, Metzger J, von Flüe M, Gass M (2020) Simplified dual time point FDG-PET/computed tomography for determining dignity of pancreatic lesions. Nucl Med Commun 41(7):682-687

38. Yamanaka M, Shinya T, Otomi Y, Kubo M, Arai Y, Toba H, Bando Y, Otsuka H, Harada M (2020) Semiquantitative assessment of fluorodeoxyglucose uptake in primary tumours on dynamic PET/computed tomography for lymph node metastasis evaluation in patients with lung cancer: a prospective study. Nucl Med Commun 41(11):1189-1198

39. Liu Z, Li M, Zuo C, Yang Z, Yang X, Ren S, Peng Y, Sun G, Shen J, Cheng C, Yang X (2021) Radiomics model of dual-time $2-\left[{ }^{18} \mathrm{~F}\right]$ FDG PET/CT imaging to distinguish between pancreatic ductal adenocarcinoma and autoimmune pancreatitis. Eur Radiol 31(9):6983-6991

40. Hwang JP, Moon JH, Kim HK, Lee MH, Lim CH, Park SB, Yoon JK, Park JM (2021) Prognostic value of metabolic parameters measured by pretreatment dual-time-point 18F-fluorodeoxyglucose positron emission tomography/computed tomography in patients with intrahepatic or perihilar cholangiocarcinoma: a STROBE study. Medicine (Baltimore) 100(21):26015

41. Cheng G, Torigian DA, Zhuang H, Alavi A (2013) When should we recommend use of dual time-point and delayed time-point imaging techniques in FDG PET? Eur J Nucl Med Mol Imaging 40:779-787

42. Morita Y, Nogami M, Sakaguchi K, Okada Y, Hirota Y, Sugawara K, Tamori Y, Zeng F, Murakami T, Ogawa W (2020) Enhanced release of glucose into the intraluminal space of the intestine associated with metformin treatment as revealed by $\left[{ }^{18} \mathrm{~F}\right]$ fluorodeoxyglucose PET-MRI. Diabetes Care 43(8):1796-1802

43. Strauss GJ (1996) Fluorine-18 deoxyglucose and false-positive results: a major problem in the diagnosis of oncological patients. Eur J Nucl Med 23:1409-1415

44. Patlak CS, Blasberg RG, Fenstermacher JD (1983) Graphical evaluation of blood-to-brain transfer constants from multiple-time uptake data. J Cereb Blood Flow Metab 3:1-7

45. Patlak CS, Blasberg RG (1985) Graphical evaluation of bloodtobrain transfer constants from multiple-time uptake data. Generalizations. J Cereb Blood Flow Metab 5:584-590

46. Karakatsanis NA, Lodge MA, Tahari AK, Zhou Y, Wahl RL, Rahmim A (2013) Dynamic whole-body PET parametric imaging: I. Concept, acquisition protocol optimization and clinical application. Phys Med Biol 58:7391-7418

47. Karakatsanis NA, Lodge MA, Zhou Y, Wahl RL, Rahmim A (2013) Dynamic whole-body PET parametric imaging: II. Taskoriented statistical estimation. Phys Med Biol 58:7419-7445

48. Fahmi G, Karakatsanis NA, Di Domenicantonio G, Garibotto V, Zaidi H (2019) Does whole-body Patlak ${ }^{18}$ F-FDG PET imaging improve lesion detectability in clinical oncology? Eur Radiol 29:4812-4821

49. Dias AH, Pedersen MF, Danielsen H, Munk OL, Gormsen LC (2021) Clinical feasibility and impact of fully automated multiparametric PET imaging using direct Patlak reconstruction: evaluation of 103 dynamic whole-body 18F-FDG PET/CT scans. Eur J Nucl Med Mol Imaging 48:837-850

50. Wang G, Qi J (2010) Acceleration of the direct reconstruction of linear parametric images using nested algorithms. Phys Med Biol 55:1505-1517

51. Karakatsanis NA, Zhou Y, Lodge MA, Casey ME, Wahl RL, Zaidi $\mathrm{H}$ et al (2015) Generalized whole-body Patlak parametric imaging for enhanced quantification in clinical PET. Phys Med Biol 60:8643-8673

52. van der Weerdt AP, Klein LJ, Visser CA, Visser FC, Lammertsma AA (2002) Use of arterialised venous instead of arterial blood for measurement of myocardial glucose metabolism during euglycaemic-hyperinsulinaemic clamping. Eur J Nucl Med Mol Imaging 29:663-669 
53. Chen K, Bandy D, Reiman E, Huang SC, Lawson M, Feng D et al (1998) Noninvasive quantification of the cerebral metabolic rate for glucose using positron emission tomography, 18F-fluoro2-deoxyglucose, the Patlak method, and an image derived input function. J Cereb Blood Flow Metab 18:716-723

54. van der Weerdt AP, Klein LJ, Boellaard R, Visser CA, Visser FC, Lammertsma AA (2001) Image-derived input functions for determination of MRGlu in cardiac (18)F-FDG PET scans. J Nucl Med 42:1622-1629

55. Takikawa S, Dhawan V, Spetsieris P, Robeson W, Chaly T, Dahl $R$ et al (1993) Noninvasive quantitative fluorodeoxyglucose PET studies with an estimated input function derived from a population-based arterial blood curve. Radiology 188:131-136

56. Shiozaki T, Sadato N, Senda M, Ishii K, Tsuchida T, Yonekura $\mathrm{Y}$ et al (2000) Noninvasive estimation of FDG input function for quantification of cerebral metabolic rate of glucose: optimization and multicenter evaluation. J Nucl Med 41:1612-1618
57. Vriens D, de Geus-Oei LF, Oyen WJ, Visser EP (2009) A curvefitting approach to estimate the arterial plasma input function for the assessment of glucose metabolic rate and response to treatment. J Nucl Med 50:1933-1939

58. Naganawa M, Gallezot JD, Shah V, Mulnix T, Young C, Dias M, Chen MK, Smith AM, Carson RE (2020) Assessment of population-based input functions for Patlak imaging of whole body dynamic ${ }^{18}$ F-FDG PET. EJNMMI Phys 7:67

Publisher's Note Springer Nature remains neutral with regard to jurisdictional claims in published maps and institutional affiliations. 